Research Paper

\title{
Prognostic Impact of ABO Blood Group on Type I Endometrial Cancer Patients- Results from Our Own and Other Studies
}

\author{
Vincenzo Dario Mandato ${ }^{* 凶}$, Federica Torricelli2*, Valentina Mastrofilippo ${ }^{3}$, Gino Ciarlini ${ }^{3}$, Debora Pirillo1, \\ Enrico Farnetti ${ }^{4}$, Loretta Fornaciari ${ }^{4}$, Bruno Casali ${ }^{4}$, Maria Carolina Gelli ${ }^{5}$, Martino Abrate ${ }^{3}$, Lorenzo \\ Aguzzoli ${ }^{3}$, Giovanni Battista La Sala ${ }^{1,6^{\#}}$, Davide Nicoli ${ }^{\#}$ \\ 1. Unit of Obstetrics and Gynaecology, Azienda Unità Sanitaria Locale - Istituto di Ricovero e Cura a Carattere Scientifico (IRCCS), Reggio Emilia, Italy; \\ 2. Laboratory of Translational Research, Azienda Unità Sanitaria Locale-Istituto di Ricovero e Cura a Carattere Scientifico (IRCCS), Reggio Emilia, Italy; \\ 3. Unit of Surgical Gynecol Oncology, Azienda Unità Sanitaria Locale - Istituto di Ricovero e Cura a Carattere Scientifico (IRCCS), Reggio Emilia, Italy; \\ 4. Laboratory of Molecular Biology, Azienda Unità Sanitaria Locale - Istituto di Ricovero e Cura a Carattere Scientifico (IRCCS), Reggio Emilia, Italy; \\ 5. Unit of Pathology, Azienda Unità Sanitaria Locale - Istituto di Ricovero e Cura a Carattere Scientifico (IRCCS), Reggio Emilia, Italy; \\ 6. Unit of Obstetrics and Gynaecology, University of Modena and Reggio Emilia, Reggio Emilia, Italy. \\ * These two authors contributed equally \\ \# These two authors contributed equally \\ $\triangle$ Corresponding author: Vincenzo Dario Mandato, Unit of Obstetrics and Gynecology, Azienda Unità Sanitaria Locale, IRCCS, Viale Risorgimento 80, 42123 \\ Reggio Emilia (Italy). Tel: +39 3494640813, Fax: +39 0522296015, e-mail: dariomandato@gmail.com. \\ (c) Ivyspring International Publisher. This is an open access article distributed under the terms of the Creative Commons Attribution (CC BY-NC) license \\ (https://creativecommons.org/licenses/by-nc/4.0/). See http://ivyspring.com/terms for full terms and conditions.
}

Received: 2017.02.06; Accepted: 2017.05.14; Published: 2017.08.23

\begin{abstract}
Objectives The $A B O$ blood group antigens were found on most epithelial cells and in secretions. In the normal endometrium there is a variable expression of histo-blood group and related antigens suggesting a hormonal regulation. A relationship between $\mathrm{ABO}$ blood groups and endometrial cancer has been investigated with contradictory results. In this study we investigated the influence of blood types on clinical and pathological characteristics of endometrial cancer patients.

Method Retrospective cohort study. Clinical and pathological data were extrapolated and their association with blood groups were assessed.

Results A total of 203 type I endometrial cancer patients were included in the final analysis. Univariate analysis indicated that a lower frequency of $G 3$ undifferentiated tumors was observed in patients with $A$ blood group $(P=0.027)$. Multivariate analysis, including also clinical features such as Age, BMI, parity, hypertension and diabetes confirmed that patients with $A$ group present a lower risk of $\mathrm{G} 3$ tumors in comparison with not $A$ patients. $(O R=0.32, P=0.011)$.

Conclusions Patients with A genotype have a lower risk to develop G3 type I endometrial cancer. $A B O$ blood group might represent a useful, easy access and cheap biomarker for patients' selection and for management personalization of endometrial cancer patients.
\end{abstract}

Key words: Endometrial cancer, Type I endometrial cancer, ABO blood group, Grading, A blood group.

\section{Introduction}

Endometrial cancer (EC) is the most common gynecological malignancy in developed countries, 319605 new cancer cases and 76160 cancer deaths worldwide were recorded in the 2012 [1-4].

Type I endometrial adenocarcinoma is the most common type, it can be classified into highly differentiated (G1), moderately differentiated (G2), or undifferentiated (G3) according to FIGO classification. Usually type I EC has a good prognosis because is a G1 tumour diagnosed at early stage [1]. Prolonged hyperestrogenism, unopposed by progesterone has a pivotal role in the pathogenesis of type I EC. 
Hyperestrogenism characterizes early menarche, late menopause, nulliparity, estrogen only hormone replacement therapy and obesity that are well known risk factors for type I EC [5,6]. Several data related to the genetic risk was also reported [7-9]. Single nucleotide polymorphisms (SNPs) of aromatase (CYP19A1) influenced susceptibility to EC, particularly among older and obese patients [7].

Some studies [10-13] have shown that the ABO blood group may influence the risk of EC.

The ABO blood group antigens were initially identified, by Landsteiner [10], as erythrocyte substances with a significance mainly ascribed to serology, but these antigens were found on most epithelial cells and in secretions [11-13].

The carbohydrate histo-blood group antigens are not primary gene products, but they are synthesized by the action of gene-encoded glycosyltransferases. The synthesis of histo-blood group antigens is stepwise, and each step is catalyzed by specific glycosyltransferases [14].

Several polymorphic genes are involved in the genetic regulation of carbohydrate synthesis which has to be taken into consideration when the expression of carbohydrates is evaluated. The pathway of the biosynthesis and the chemical structure of the antigens explains the interrelationships between the $\mathrm{ABO}$ and $\mathrm{H}$ systems [15].

This synthesis correlates with embryonic development and cellular differentiation [16]. The expression of histo-blood group antigens varies in this way from cell to cell and from organ to organ. The blood group antigens represent the terminal part of an oligosaccharide chain linked to proteins or lipids. The antigen determinants may be carried on many different core saccharide structures, and the general phenotype of the $\mathrm{ABH}$ epitopes is uninfluenced by the carbohydrates that carry them [16-17].

In the normal endometrium there is a variable expression of histo-blood groups and related antigens suggesting a hormonal regulation of glycosyltransferase activity. The expression of the A/B transferase proteins in human endometrial epithelial cells was shown to correlate with the level of oestradiol in serum [18] and is influenced by the secretor status [19]. Moreover, ABO carbohydrates seem to strictly regulate the adhesion and implantation of the blastocyst process [20].

A relationship between $\mathrm{ABO}$ blood groups with $\mathrm{EC}$ has been investigated with contradictory results (Table 1). In a study, A blood group had the highest frequency between the women with EC in the reproductive age. $\mathrm{O}$ phenotype group was the most frequent in case of menopause and post-menopause women with EC [21]. On contrary in another study no statistically significant correlations were obtained for $\mathrm{EC}$ and ABO blood groups [22].

In this study we investigated the influence of blood types on EC patients treated at our research institute, particularly we evaluated the impact of blood types on clinical and pathological characteristics.

\section{Methods}

\section{Patient samples}

The study was designed following the Strengthening the Reporting of Observational Studies in Epidemiology (STROBE) statement [23]. The Local Ethical Committee approved the study design and all patients provided written informed consent to use personal non-sensitive data at hospital admission.

Clinical charts of EC patients treated and followed at the IRCCS - Santa Maria Nuova Hospital of Reggio Emilia (Italy) from 1997 to 2016 were checked for inclusion and exclusion criteria.

Clinical, pathological and genetic data were recorded in an electronic separate, anonymous, password-protected database. All relevant data were extrapolated and used for final analysis.

Patients with histological diagnosis of type I EC who received upfront adequate surgery treatment were electively included in the protocol study. Exclusion criteria were: histological diagnosis of non type I EC, inadequate EC management according to internal and international guidelines [24, 25], neoadjuvant chemotherapy performed before surgery, an age less than 18 years, non-Caucasian ancestry, a follow-up length less than of 6 months, inadequate follow-up according to internal guidelines, absence of written informed consent, diagnosis of a previous or concurrent cancer(s) and unavailable follow-up data.

An "adequate" management was considered as follows: total extrafascial hysterectomy (TEH) with bilateral salpingo-oophorectomy (BSO) was the standard staging procedure; whereas radical hysterectomy (RH) was performed only in stage II EC patients with gross cervical involvement; pelvic with/without paraaortic lymph node dissection were performed in case of myometrial invasion greater than 50 percent, large tumor ( $>2 \mathrm{~cm}$ in diameter) or filling the endometrial cavity. Vaginal brachytherapy alone was administered to the patients at stage IA G3 and IB G1 or G2 without negative prognostic factors. External beam radiotherapy plus vaginal brachytherapy was administered to the patients at stage IA G3 and IB G1 and G2 with negative prognostic factors, and to the patients at stage IB G3 
and to all the patients at stage II, III and IV. Chemotherapy was administered to the patients at stage III C and IV. In all cases, chemoradiotherapy consisted of paclitaxel $175 \mathrm{mg} / \mathrm{m}^{2}(\mathrm{P})$ and carboplatin AUC5 (C) on day 1 every three weeks, for a total of four to six cycles, and it was followed by external pelvic radiation therapy $(1.8 \mathrm{~Gy} / \mathrm{d}, \mathrm{d} 1-5)$ at a total dose of 45 Gy plus vaginal brachytherapy $(3 \times 5$ Gy) [24, 26].

A follow-up was defined "adequate" in case of adherence to the following schedule: type I EC at stage IA and grading G1/G2 - physical and gynecological examination, and transvaginal ultrasound every 6 months for the first 2 years, and then every 12 months for at least 3 years; type I EC at stage IB and/or any grading G3 tumor - physical and gynecological examination, and transvaginal ultrasound every 6 months for the first 5 years. Further investigations such as abdominal ultrasound, chest X-ray, computed tomography scan, and serum CA 125 levels were performed if clinically indicated.

The same pathologist with long-time expertise in gynecological oncology reviewed all the histological samples in order to confirm formally the diagnosis.

\section{Statistical analysis}

For statistical analysis, $\mathrm{R}$ statistical software package version 2.15.1 ( $\mathrm{R}$ foundation for Statistical Computing, Vienna, Austria) was used.

Fisher's exact test and generalized linear models were used to investigate univariate and multivariate association of blood groups with clinical and pathological parameters.

Table 1. ABO blood groups and endometrial cancers: results reported in literature

\begin{tabular}{|c|c|c|c|c|c|c|c|c|c|c|c|c|c|c|c|c|}
\hline Year & $\begin{array}{l}\text { Author } \\
\text { (reference) }\end{array}$ & Ethnicity & $\begin{array}{l}\text { Sample } \\
\text { Size }\end{array}$ & Age (n) & BMI (n) & $\begin{array}{l}\text { FIGO } \\
\text { Stage } \\
(\mathbf{n})\end{array}$ & $\begin{array}{l}\text { Gradin } \\
\mathrm{g}(\mathrm{n})\end{array}$ & $\begin{array}{l}0 \% \\
\text { (n) }\end{array}$ & $\begin{array}{l}P \\
\text { value }\end{array}$ & A \% (n) & $\begin{array}{l}P \\
\text { value }\end{array}$ & B \% (n) & $\begin{array}{l}P \\
\text { value }\end{array}$ & $\begin{array}{l}\mathrm{AB} \% \\
\text { (n) }\end{array}$ & $\begin{array}{l}P \\
\text { value }\end{array}$ & Conclusion \\
\hline \multirow[t]{3}{*}{2014} & $\begin{array}{l}\text { Nakashidze I } \\
\text { et al. (21) }\end{array}$ & Georgia & 60 & $\begin{array}{l}\text { Reproductive } \\
\text { Age (20) }\end{array}$ & - & - & - & $\begin{array}{l}20 \% \\
(4)\end{array}$ & $<0.05$ & $65 \%(13)$ & $<0.05$ & $10 \%(2)$ & $<0.05$ & $5 \%(1)$ & $<0.05$ & $\begin{array}{l}\text { A group is the } \\
\text { most common in } \\
\text { reproductive age }\end{array}$ \\
\hline & & & & Menopause (20) & & & & $\begin{array}{l}55 \% \\
(11)\end{array}$ & & $40 \%(8)$ & & $5 \%(1)$ & & $0 \%(0)$ & & $\begin{array}{l}\mathbf{0} \text { group is the } \\
\text { most common in }\end{array}$ \\
\hline & & & & $\begin{array}{l}\text { Postmenopause } \\
(20)\end{array}$ & & & & $\begin{array}{l}60 \% \\
(12)\end{array}$ & & $30 \%(6)$ & & $5 \%(1)$ & & $5 \%(1)$ & & $\begin{array}{l}\text { menopause and } \\
\text { post menopause } \\
\text { age }\end{array}$ \\
\hline \multirow[t]{2}{*}{2012} & $\begin{array}{l}\text { Yuzhalin AE } \\
\text { et al. (22) }\end{array}$ & Siberia & 440 & $\begin{array}{l}\text { Premenopause } \\
(102)\end{array}$ & - & - & - & $\begin{array}{l}31.4 \% \\
(32)\end{array}$ & - & $\begin{array}{l}36.3 \% \\
(37)\end{array}$ & 0.495 & $\begin{array}{l}24.5 \% \\
(25)\end{array}$ & 0.435 & $\begin{array}{l}7.8 \% \\
(8)\end{array}$ & 0.906 & $\begin{array}{l}\text { No statistically } \\
\text { significant } \\
\text { correlation }\end{array}$ \\
\hline & & & & $\begin{array}{l}\text { Postmenopause } \\
(338)\end{array}$ & & & & $\begin{array}{l}35.5 \% \\
(120)\end{array}$ & - & $\begin{array}{l}37 \% \\
(125)\end{array}$ & 0.639 & $\begin{array}{l}19.8 \% \\
(67)\end{array}$ & 0.409 & $\begin{array}{l}7.7 \% \\
(26)\end{array}$ & 0.659 & \\
\hline \multirow[t]{3}{*}{1995} & $\begin{array}{l}\text { Marinaccio M } \\
\text { et al. (29) }\end{array}$ & Italy & 237 & - & - & $\begin{array}{l}\text { I } \\
(119)\end{array}$ & - & $\begin{array}{l}23.5 \% \\
(28)\end{array}$ & 0.001 & $\begin{array}{l}56.3 \% \\
(67)\end{array}$ & - & $\begin{array}{l}16.0 \% \\
(19)\end{array}$ & - & $\begin{array}{l}4.2 \% \\
(5)\end{array}$ & - & $\begin{array}{l}\text { A group is the } \\
\text { most common in } \\
\text { the stage I }\end{array}$ \\
\hline & & & & & & II (68) & & $\begin{array}{l}66.2 \% \\
(45)\end{array}$ & - & $\begin{array}{l}25.0 \% \\
(17)\end{array}$ & - & $8.8 \%(6)$ & - & - & - & $\begin{array}{l}0 \text { group is the } \\
\text { most common in } \\
\text { the stage II }\end{array}$ \\
\hline & & & & & & $\begin{array}{l}\text { NA } \\
(50)\end{array}$ & & $\begin{array}{l}24.0 \% \\
(12)\end{array}$ & - & $58 \%(29)$ & - & $18 \%(9)$ & - & - & - & \\
\hline 2011 & $\begin{array}{l}\text { Xu W et al. } \\
\text { (30) }\end{array}$ & China & 1204 & 54,3 (mean) & $\begin{array}{l}25,7 \\
(\text { mean) }\end{array}$ & - & - & $\begin{array}{l}323 \\
(26 \%)\end{array}$ & 0.001 & $\begin{array}{l}355 \\
(29,4 \%)\end{array}$ & 0.001 & $\begin{array}{l}265 \\
(22.0 \%)\end{array}$ & 0.001 & $\begin{array}{l}126 \\
(126 / \\
1204)\end{array}$ & 0.001 & $\begin{array}{l}\text { A group is the } \\
\text { most common in } \\
\text { woman with } \\
\text { endometrial } \\
\text { cancer }\end{array}$ \\
\hline \multirow[t]{10}{*}{2017} & $\begin{array}{l}\text { Mandato VD } \\
\text { et al. }\end{array}$ & Italy & 203 & $\leq 64(104)$ & & & & $\begin{array}{l}45.2 \% \\
(33)\end{array}$ & - & $\begin{array}{l}56.7 \% \\
(59)\end{array}$ & 0.132 & $\begin{array}{l}42.9 \% \\
(9)\end{array}$ & 0.849 & $\begin{array}{l}60 \% \\
(3)\end{array}$ & 0.526 & $\begin{array}{l}\text { No statistically } \\
\text { significant }\end{array}$ \\
\hline & & & & > 64 (99) & & & & $\begin{array}{l}54.8 \% \\
(40)\end{array}$ & & $\begin{array}{l}43.3 \% \\
(45)\end{array}$ & & $\begin{array}{l}57.1 \% \\
(12)\end{array}$ & & $\begin{array}{l}40 \% \\
(2)\end{array}$ & & $\begin{array}{l}\text { correlation } \\
\text { between blood } \\
\text { group and age }\end{array}$ \\
\hline & & & & & $\begin{array}{l}\leq 28 \\
(100)\end{array}$ & & & $\begin{array}{l}57.1 \% \\
(40)\end{array}$ & - & $\begin{array}{l}46.1 \% \\
(48)\end{array}$ & 0.174 & $40 \%(8)$ & 0.180 & $\begin{array}{l}805 \\
(4)\end{array}$ & 0.337 & $\begin{array}{l}\text { No statistically } \\
\text { significant }\end{array}$ \\
\hline & & & & & $>28(98)$ & & & $\begin{array}{l}42.9 \% \\
(30)\end{array}$ & & $\begin{array}{l}53.4 \% \\
(55)\end{array}$ & & $60 \%(12)$ & & $\begin{array}{l}20 \% \\
(2)\end{array}$ & & $\begin{array}{l}\text { correlation } \\
\text { between blood }\end{array}$ \\
\hline & & & & & NA (5) & & & 3 & & 1 & & 1 & & 0 & & group and BMI \\
\hline & & & & & & $\begin{array}{l}\text { I-II } \\
(187)\end{array}$ & & $\begin{array}{l}90.4 \% \\
(66)\end{array}$ & - & $\begin{array}{l}92.3 \% \\
(96)\end{array}$ & 0.656 & $\begin{array}{l}95.2 \% \\
(20)\end{array}$ & 0.494 & $\begin{array}{l}100 \% \\
(5)\end{array}$ & 0.989 & $\begin{array}{l}\text { No statistically } \\
\text { significant } \\
\text { correlation } \\
\text { between blood } \\
\text { group and FIGO } \\
\text { stage }\end{array}$ \\
\hline & & & & & & $\begin{array}{l}\text { III-IV } \\
(16)\end{array}$ & & $\begin{array}{l}9.6 \% \\
(7)\end{array}$ & & $7.7 \%(8)$ & & $4.8 \%(1)$ & & 0 & & \\
\hline & & & & & & & G1 (88) & $\begin{array}{l}37.0 \% \\
(27)\end{array}$ & - & $\begin{array}{l}49.9 \% \\
(51)\end{array}$ & - & $28.6 \%(6)$ & - & $\begin{array}{l}80 \% \\
(4)\end{array}$ & - & \multirow{3}{*}{$\begin{array}{l}\text { A group presents } \\
\text { a lower risk of } G 3 \\
\text { endometrial } \\
\text { cancer in } \\
\text { comparison with } \\
\text { not A group }\end{array}$} \\
\hline & & & & & & & G2 (81) & $\begin{array}{l}41.1 \% \\
(30)\end{array}$ & & $\begin{array}{l}40.4 \% \\
(42)\end{array}$ & 0.375 & $38.1 \%(8)$ & 0.762 & $\begin{array}{l}20 \% \\
(1)\end{array}$ & 0.194 & \\
\hline & & & & & & & G3 (34) & $\begin{array}{l}21.9 \% \\
(16)\end{array}$ & & $\begin{array}{l}10.6 \% \\
(11)\end{array}$ & 0.027 & $\begin{array}{l}33.3 \% \\
(7)\end{array}$ & 0.289 & 0 & 0.989 & \\
\hline
\end{tabular}


Overall survival (OS) was computed as the time period from the date of surgery to either the date of death or last follow up, whichever occurred first. Disease free survival (DFS) was computed as the time period from the date of surgery to either the date of diagnosis of recurrence or last follow up, whichever occurred first. The effects of genotypes on OS and DFS were evaluated using Cox regression hazard model and results were presented as hazard ratio (HR).

Significant statements referred $\mathrm{P}$ values lower than 0.05 .

\section{Results}

After patients' selection for the inclusion and exclusion criteria, a total of 203 EC patients were studied and included in the final analysis. Total extrafascial hysterectomy was performed in 140 $(69.0 \%)$ patients, whereas radical hysterectomy was performed in 45 (22.1\%) patients. Salpingo-oophorectomy was performed in 195 (6.1\%) patients. Omentectomy and appendicectomy were performed in $19(9.4 \%)$ and $4(2.0 \%)$ patients, respectively. One hundred thirty eight (68.0\%) patients received pelvic lymphadenectomy and only $13(6.4 \%)$ patients received lombo-aortic lymphadenectomy. In all patients a complete resection of the disease was obtained.
During a median follow-up time of 57 months (range, 7 to 151 months) 16 patients had a recurrence and 11 patients died because of the cancer.

In our population there were 73 patients with blood group $0(36.0 \%), 104$ with A $(51.0 \%), 21$ with B $(10.3 \%)$ and 5 with $\mathrm{AB}(2.5 \%)$.

In table 2 blood groups $\mathrm{AB} 0$ were analyzed in association with clinical and pathological characteristics of EC patients. Interestingly a significant association was observed between tumor grading and patients' blood group, in particular a lower frequency of G3 undifferentiated tumors was observed in patients with A blood group $(\mathrm{P}=0.027)$ (Figure 1).

In table 3 the association was further investigated in multivariate analysis, including also clinical features such as Age, BMI, parity, hypertension and diabetes. Blood groups were clustered to investigate in particular the effect of $\mathrm{O}$ and A group on risk of high grade tumor. The analysis confirmed that patients with A group present a lower risk of G3 EC tumors in comparison with not A patients $(\mathrm{OR}=0.32, \mathrm{P}=0.011)$ (Figure 2).

No association of blood groups with OS and DFS of EC patients was observed in our study population (Table 4).

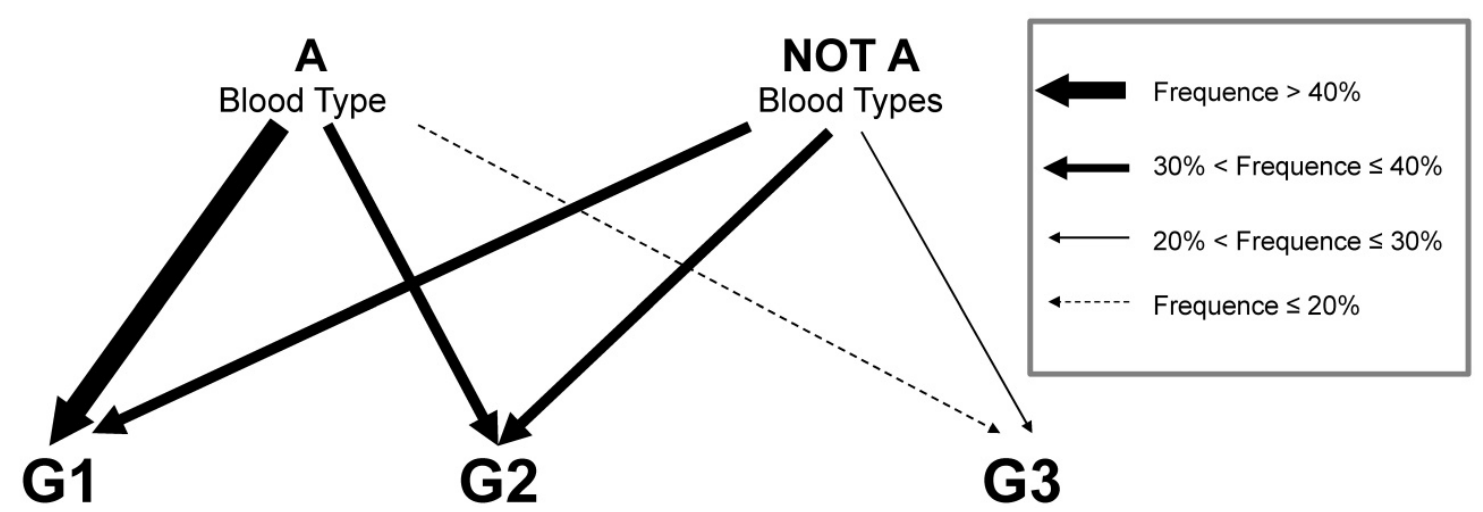

Figure 1. Representation in diagram of A and not A blood groups effect on type $1 \mathrm{EC}$. As indicated in legend arrows thickness express the range of frequency of each grade in the 2 group of patients.

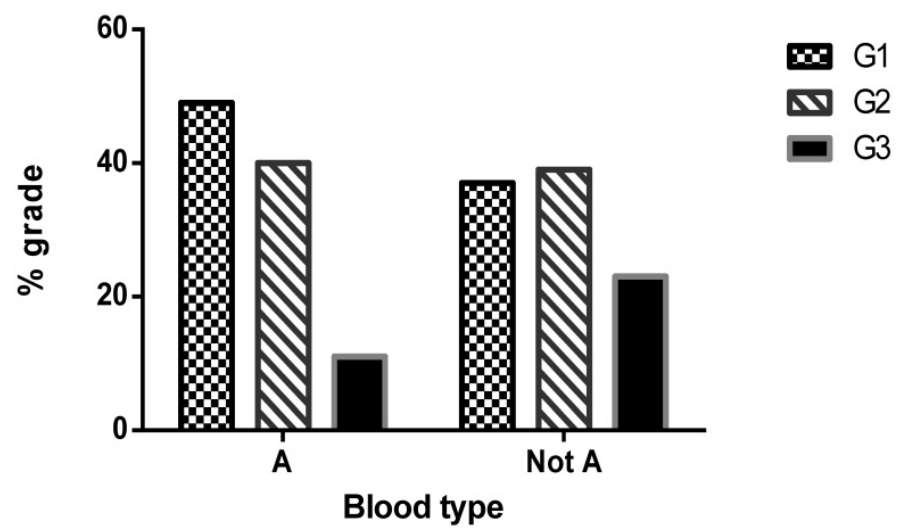

Figure 2. Histogram of type $1 \mathrm{EC}$ grades frequencies in $A$ and not $A$ population. 
Table 2. Association study of blood groups $A B 0$ with clinical and pathological features of the type I endometrial cancer.

\begin{tabular}{|c|c|c|c|c|c|c|c|c|c|}
\hline Features & $\begin{array}{l}\text { Patients }(\mathrm{n} \%) \\
(\mathrm{N}=203,100 \%)\end{array}$ & $\begin{array}{l}0(\mathrm{n} \%) \\
(\mathrm{N}=73,100 \%)\end{array}$ & $\begin{array}{l}P \\
\text { value }\end{array}$ & $\begin{array}{l}\mathbf{A}(\mathrm{n} \%) \\
(\mathrm{N}=104,100 \%)\end{array}$ & $\begin{array}{l}P \\
\text { value }\end{array}$ & $\begin{array}{l}\text { B (n\%) } \\
(\mathrm{N}=21,100 \%)\end{array}$ & $\begin{array}{l}P \\
\text { value }\end{array}$ & $\begin{array}{l}\mathbf{A B}(\mathrm{n} \%) \\
(\mathrm{N}=5,100 \%)\end{array}$ & P value \\
\hline \multicolumn{10}{|c|}{ 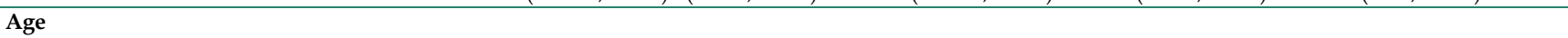 } \\
\hline$\leq 64$ & $104(51.2)$ & $33(45.2)$ & & $59(56.7)$ & & 9 (42.9) & & $3(60.0)$ & \\
\hline$>64$ & $99(48.8)$ & $40(54.8)$ & & $45(43.3)$ & 0.132 & $12(57.1)$ & 0.849 & $2(40.0)$ & 0.526 \\
\hline \multicolumn{10}{|l|}{ BMI } \\
\hline$\leq 28$ & $100(50.5)$ & $40(57.1)$ & & 48 (46.6) & & $8(40.0)$ & & $4(80.0)$ & \\
\hline$>28$ & $98(49.5)$ & $30(42.9)$ & & $55(53.4)$ & 0.174 & $12(60.0)$ & 0.180 & $1(20.0)$ & 0.337 \\
\hline$N A^{*}$ & 5 & 3 & & 1 & & 1 & & 0 & \\
\hline \multicolumn{10}{|l|}{ Parity } \\
\hline No & $33(16.4)$ & $11(15.3)$ & & $16(15.5)$ & & $5(23.8)$ & & $1(20.0)$ & \\
\hline Yes & $168(83.6)$ & $61(84.7)$ & & 87 (84.5) & 0.963 & $16(76.2)$ & 0.366 & $4(80.0)$ & 0.779 \\
\hline$N A^{*}$ & 2 & 1 & & 1 & & 0 & & 0 & \\
\hline \multicolumn{10}{|l|}{ Hypertension } \\
\hline No & 83 (41.3) & $36(50.0)$ & & 37 (35.9) & & $6(28.6)$ & & $4(80.0)$ & \\
\hline Yes & $118(58.7)$ & $36(50.0)$ & & $66(64.1)$ & 0.064 & 15 (71.4) & 0.088 & $1(20.0)$ & 0.225 \\
\hline$N A^{*}$ & 2 & 1 & & 1 & & 0 & & 0 & \\
\hline \multicolumn{10}{|l|}{ Diabetes } \\
\hline No & 159 (79.1) & $62(86.1)$ & & 77 (74.7) & & 15 (71.4) & & $5(100.0)$ & \\
\hline Yes & $42(20.9)$ & $10(13.9)$ & & $26(25.3)$ & 0.071 & $6(28.6)$ & 0.124 & $0(0.0)$ & 0.989 \\
\hline$N A^{*}$ & 2 & 1 & & 1 & & 0 & & 0 & \\
\hline \multicolumn{10}{|l|}{ Figo Stage } \\
\hline$I-I I$ & $187(92.1)$ & $66(90.4)$ & & $96(92.3)$ & & $20(95.2)$ & & $5(100.0)$ & \\
\hline$I I I-I V$ & $16(7.9)$ & $7(9.6)$ & & $8(7.7)$ & 0.656 & $1(4.8)$ & 0.494 & $0(0.0)$ & 0.989 \\
\hline \multicolumn{10}{|l|}{ Grading } \\
\hline G1 & 88 (43.3) & $27(37.0)$ & & $51(49.0)$ & & $6(28.6)$ & & $4(80.0)$ & \\
\hline G2 & 81 (39.9) & $30(41.1)$ & & $42(40.4)$ & 0.375 & $8(38.1)$ & 0.762 & $1(20.0)$ & 0.194 \\
\hline G3 & $34(16.8)$ & $16(21.9)$ & & $11(10.6)$ & 0.027 & $7(33.3)$ & 0.289 & $0(0.0)$ & 0.989 \\
\hline \multicolumn{10}{|l|}{ Adjuvant Treatment } \\
\hline No & $141(69.5)$ & $50(68.5)$ & & $76(73.1)$ & & $10(47.6)$ & & $5(100.0)$ & \\
\hline Yes & $62(30.5)$ & $23(31.5)$ & & $28(26.9)$ & 0.508 & $11(52.4)$ & 0.084 & $0(0.0)$ & 0.988 \\
\hline \multicolumn{10}{|l|}{ Death } \\
\hline No & $178(87.7)$ & $64(87.7)$ & & $92(88.5)$ & & $18(85.7)$ & & $4(80.0)$ & \\
\hline Yes & $25(12.3)$ & $9(12.3)$ & & $12(11.5)$ & 0.873 & $3(14.3)$ & 0.813 & $1(20.0)$ & 0.624 \\
\hline \multicolumn{10}{|l|}{ Death because of the tumor } \\
\hline No & 192 & $70(95.9)$ & & $97(93.3)$ & & $20(95.2)$ & & $5(100.0)$ & \\
\hline Yes & 11 & $3(4.1)$ & & $7(6.7)$ & 0.462 & $1(4.8)$ & 0.896 & $0(0.0)$ & 0.993 \\
\hline \multicolumn{10}{|l|}{ Recurrence } \\
\hline No & 186 & $69(94.5)$ & & $94(90.4)$ & & $19(90.5)$ & & $4(80.0)$ & \\
\hline Yes & 17 & $4(5.5)$ & & $10(9.6)$ & 0.322 & $2(9.5)$ & 0.509 & $1(20.0)$ & 0.235 \\
\hline Lymph node Metastasis (132 Lymphadenectomy) & 132 & 53 & & 62 & & 13 & & 4 & \\
\hline No & 121 & $48(90.6)$ & & $57(93.4)$ & & $12(100.0)$ & & $4(100.0)$ & \\
\hline Yes & 9 & $5(9.4)$ & & $4(6.6)$ & 0.572 & $0(0.0)$ & 0.993 & $0(0.0)$ & 0.996 \\
\hline$N A^{*}$ & 2 & 0 & & 1 & & 1 & & 0 & \\
\hline
\end{tabular}

\section{Discussion}

A relationship between $\mathrm{ABO}$ blood groups and EC cancer risk has been investigated in several studies. However, to our knowledge no study reported a significant favourable association between A genotype and tumor grading. Particularly, patients with A genotype have a lower risk $(P=0.011)$ to develop G3 EC in comparison with patients with other blood group combinations.

Previous studies reported that the A blood group was associated with high risk of EC [29] particularly in reproductive age [21].

A significant dose response relationship was observed for EC risk and level of antigen A. The positive association of blood type A with cancer risk was observed regardless of menopausal status, body mass index, oral contraceptive use, or family cancer history suggesting that $\mathrm{ABO}$ blood type may be involved in the development of EC [30].

On contrary, the $\mathrm{O}$ blood group was associated with high risk of EC in menopause and post-menopause women [21] and in another study no statistically significant correlations were obtained for $\mathrm{EC}$ and $\mathrm{ABO}$ [22].

Several mechanisms for the association of the $\mathrm{ABO}$ blood type with cancer risk have been proposed, including inflammation, immune surveillance for malignant cells, intercellular adhesion, and membrane signaling [31].

$\mathrm{ABO}$ antigens may interfere with cell adhesion, cell signaling and immune surveillance by altered levels of tumor necrosis factor-a, E-selectin and P-selectin [32, 33]. 
Table 3. Multivariate analysis of association between patients' blood groups $A B 0$ and clinical features and grade of type 1 endometrial cancer. Blood groups were also clustered to investigate in particular the effect of $O$ and $A$ group on tumor grades frequencies.

\begin{tabular}{|c|c|c|c|c|c|c|c|c|c|}
\hline Features & & $\begin{array}{l}\text { Patients } \\
(\mathrm{N}=203,100 \%)\end{array}$ & $\begin{array}{l}\text { Grade G1 } \\
(\mathrm{N}=88)(\mathrm{n} \%)\end{array}$ & $\begin{array}{l}\text { Grade G2 } \\
(\mathrm{N}=81)(\mathrm{n} \%)\end{array}$ & $\begin{array}{l}\text { Adjusted OR } \\
(95 \% \mathrm{CI})\end{array}$ & $\begin{array}{l}\text { Adjusted } \\
\text { P value }\end{array}$ & $\begin{array}{l}\text { Grade G3 } \\
(\mathrm{N}=34)(\mathrm{n} \%)\end{array}$ & $\begin{array}{l}\text { Adjusted OR } \\
(95 \% \mathrm{CI})\end{array}$ & $\begin{array}{l}\text { Adjusted } \\
\text { P value }\end{array}$ \\
\hline \multirow[t]{4}{*}{ Blood group } & 0 & 73 & $27(37.0)$ & $30(41.0)$ & - & - & $16(21.9)$ & - & - \\
\hline & $\mathrm{A}$ & 104 & $51(49.0)$ & $42(40.4)$ & $0.73(0.36-1.47)$ & 0.378 & $11(10.6)$ & $0.32(0.11-0.86)$ & 0.028 \\
\hline & B & 21 & $6(28.6)$ & $8(38.1)$ & $1.38(0.38-5.41)$ & 0.628 & $7(33.3)$ & $2.23(0.58-9.18)$ & 0.249 \\
\hline & $\mathrm{AB}$ & 5 & $4(80.0)$ & $1(20.0)$ & $0.25(0.01-1.93)$ & 0.237 & $0(0.0)$ & - & 0.990 \\
\hline \multirow[t]{2}{*}{ Age (years) } & $\leq 64$ & 104 & $50(48.1)$ & $36(34.6)$ & - & - & $18(17.3)$ & & \\
\hline & $>64$ & 99 & $38(38.4)$ & $45(45.4)$ & $1.42(0.73-2.77)$ & 0.301 & $16(16.2)$ & $0.84(0.31-2.22)$ & 0.734 \\
\hline \multirow[t]{3}{*}{ BMI } & $\leq 28$ & 100 & $47(47.0)$ & $38(38.0)$ & - & - & $15(15.0)$ & - & - \\
\hline & $>28$ & 98 & $38(38.8)$ & $42(42.9)$ & $1.35(0.67-2.71)$ & 0.398 & $18(18.4)$ & $1.30(0.51-3.35)$ & 0.588 \\
\hline & NA & 5 & & & & & & & \\
\hline \multirow[t]{3}{*}{ Parity } & No & 33 & $17(51.5)$ & $7(21.2)$ & - & - & $9(27.3)$ & - & - \\
\hline & Yes & 168 & $69(41.1)$ & $74(44.0)$ & $2.76(1.07-7.82)$ & 0.042 & $25(14.9)$ & $0.78(0.29-2.23)$ & 0.632 \\
\hline & NA & 2 & & & & & & & \\
\hline \multirow[t]{3}{*}{ Hypertension } & No & 83 & $38(45.8)$ & $30(36.1)$ & & & $15(18.1)$ & & \\
\hline & Yes & 118 & $48(40.7)$ & $51(43.2)$ & $0.98(0.48-1.98)$ & 0.951 & $19(16.1)$ & $0.99(0.37-2.71)$ & 0.998 \\
\hline & NA & 2 & & & & & & & \\
\hline \multirow[t]{3}{*}{ Diabetes } & No & 159 & $70(44.0)$ & $63(39.6)$ & & & $26(16.3)$ & & \\
\hline & Yes & 42 & $16(38.1)$ & $18(42.9)$ & $1.17(0.50-2.72)$ & 0.719 & $8(19.0)$ & $1.67(0.49-5.66)$ & 0.407 \\
\hline & NA & 2 & & & & & & & \\
\hline \multicolumn{10}{|c|}{ Clustered Blood groups } \\
\hline 0 & & 73 & $27(37.0)$ & $30(41.1)$ & & & $16(21.9)$ & & \\
\hline Not 0 & & 130 & $61(46.9)$ & $51(39.2)$ & $0.76(0.39-1.50)$ & 0.434 & $18(13.9)$ & $0.48(0.20-1.12)$ & 0.089 \\
\hline Not A & & 99 & $37(37.4)$ & $39(39.4)$ & & & $23(23.2)$ & & \\
\hline A & & 104 & $51(49.0)$ & $42(40.4)$ & $0.76(0.40-1.44)$ & 0.393 & $11(10.6)$ & $0.32(0.13-0.76)$ & 0.011 \\
\hline
\end{tabular}

NA=Not Available; OR=Odd Ratio

Table 4. Cox model evaluation of the effects of different blood groups on overall survival and disease free survival in type 1 endometrial cancer patients.

\begin{tabular}{|c|c|c|c|c|c|c|c|}
\hline \multirow[t]{2}{*}{ Features } & \multirow{2}{*}{$\begin{array}{l}\text { Patients } \\
(\mathrm{N}=203,100 \%)\end{array}$} & \multicolumn{3}{|c|}{ Overall Survival } & \multicolumn{3}{|c|}{ Disease free survival } \\
\hline & & $\begin{array}{l}\text { \# Death } \\
(\mathrm{N}=11)\end{array}$ & HR $(95 \%$ CI $)$ & P value & $\begin{array}{l}\text { \# Recurrence } \\
(\mathrm{N}=17)\end{array}$ & $\mathrm{HR}(95 \% \mathrm{CI})$ & $P$ value \\
\hline \multicolumn{8}{|c|}{ Blood groups } \\
\hline 0 & 73 & $3(4.1)$ & - & - & $4(5.8)$ & - & \\
\hline$A$ & 104 & $7(6.7)$ & $1.85(0.48-7.16)$ & 0.373 & $10(9.6)$ & $1.86(0.58-5.95)$ & 0.293 \\
\hline$B$ & 21 & $1(4.7)$ & $1.26(0.13-12.07)$ & 0.844 & $2(9.5)$ & $1.96(0.36-10.73)$ & 0.436 \\
\hline$A B$ & 5 & $0(0.0)$ & - & 0.999 & $1(20.0)$ & $4.63(0.51-41.82)$ & 0.172 \\
\hline \multicolumn{8}{|c|}{ Clustered Blood groups } \\
\hline 0 & 73 & $3(4.1)$ & - & - & $4(5.5)$ & - & - \\
\hline Not 0 & 130 & $8(6.2)$ & $0.60(0.44-6.30)$ & 0.450 & $13(10.0)$ & $1.97(0.64-6.04)$ & 0.236 \\
\hline Not A & 99 & $4(4.0)$ & - & - & $7(7.1)$ & & \\
\hline$A$ & 104 & $7(6.7)$ & $1.85(0.54-6.34)$ & 0.325 & $10(9.6)$ & $1.39(0.53-3.66) \backslash$ & 0.501 \\
\hline
\end{tabular}

HR=Hazard Ratio

The ABO gene is located on chromosome 9q34. $\mathrm{ABO}$ gene polymorphism has been implicated in susceptibility to several cancers across different populations, but a susceptibility to EC has not been reported [34].

This gene encodes for glycosyltransferases, which catalyze the step-by step transfer of single sugars to the $\mathrm{H}$ antigen to form the $\mathrm{A}$ and $\mathrm{B}$ antigen [35].

The peripheral part of the carbohydrates in the cell surface is strongly immunogenic, many carbohydrates, including the blood group $\mathrm{ABO}$ antigens, were initially identified as cell surface antigens [14].

Blood group $\mathrm{O}$ persons, who do not have the A and $B$ gene coded glycosyltransferase, express a fucosylated variant (Ley) of the precursor structure [36]. The lack of expression of blood group antigens in tumours is correlated with lack of presence of the blood group coded glycosyltransferase [37]. Aberrant glycosylation patterns are a hallmark of cancer development and progression [38, 39] and aberrant glycosylation occurs early during oncogenic transformation and may represent a key event in invasion and metastasis.

Loss or reduction of A and B epitopes in human cancers is well documented, and loss of A and B antigens is correlated with the degree of malignancy and metastatic potential in EC, lung, bladder and oral carcinomas [33, 38, 40].

In vitro studies have demonstrated that loss or addition of a single glycosyl residue may affect tumor 
cell motility by altering glycosylation of integrin receptors and their interaction with 1 integrin. This may explain the observed correlation between glycosylation and prognosis [41].

Expression of A/B antigens in tumors is directly correlated with A and B glycosyltransferase activity [37]. An antigen negative tumors have reduced levels of $\mathrm{ABO}$ transcript as compared to $\mathrm{A}$ antigen positive tumors [38]. The regulatory mechanism of $\mathrm{ABO}$ gene transcription presents two promoter regions $[42,43]$.

Expression of the $\mathrm{ABO}$ gene in epithelial and erythroid cells lines was shown to be dependent on the methylation status of the proximal constitutive promoter encoding most of the $\mathrm{ABO}$ transcripts, as an inverse relationship was found between promoter hypermethylation and $\mathrm{ABO}$ gene expression [42].

Hence, poorly differentiated tumour contained high amounts of fully methylated alleles. The levels of DNA methylation have been shown to increase with the degree of malignancy. Hypermethylation in hyperplastic or dysplastic epithelium is found, it may therefore be an early sign of malignant transformation [38].

Both non-invasive (preneoplastic) endometrial lesions and EC show changes in histo-blood group phenotype, when this is compared with that of normal endometrium. In EC, the changes in histo-blood group phenotype are qualitatively influenced by the genetic status in terms of the $A B O$, Lewis and the $\mathrm{ABH}$-secretor status predominantly. The malignant phenotype shows some resemblance to the luteal phase phenotype indicating that the responsible changes in glycosyltransferases and substrate levels may be alike. In both normal and malignant endometrial cells, the expression of A/B transferase protein is confined to endometrium from blood group A/B individuals and relate to serum estradiol levels. Loss of A/B transferase protein seems to be a late event in endometrial carcinogenesis, whereas the other changes in glycosyltransferaseactivity responsible for the observed changes in histo-blood group phenotype seem to take place in premalignant endometrial cells [44]. At the precancerous stage, $\mathrm{ABH}$ antigens are highly expressed on epithelial cells. They participate in the phenomenon of apoptosis resistance. This would facilitate both cancerogenesis and immune escape. At more advanced stages of tumor progression, tumor cells that have lost A and B antigens would be potentially more metastatic since these antigens inhibit cell motility. Similarly, overexpression of sialyl-Le ${ }^{x}$ and sialyl-Le ${ }^{a}$ would increase metastatic potential by allowing adhesion to the vascular endothelium. In addition, at intermediate stages, the angiogenic and procoagulating activities of the $\mathrm{H}$ and
Le $^{y}$ antigens would favor tumor development. Thus antigens of this family could have either a deleterious or a favorable impact on evolution of the disease. The presence of $\mathrm{ABH}$ antigen at early stages would be deleterious, while it would become favorable at latter stages by inhibiting cell motility on the one hand and synthesis of sialyl-Lewis antigens through the competition between al, 2fucosyltrasnferase and a 2.3sialyltransferase on the other [45].

In order to explore that influence, our population was studied according to well known risk factors such as age, body mass index (BMI), parity, diabetes, hypertension but at multivariate analysis, only $\mathrm{AB} 0$ blood group showed an influence on EC grade.

Tumor grade represents a well-established risk factor, according to tumor grade staging procedure such as systematic lymphadenectomy, and adjuvant treatment are performed or not [26]. Recently also rs5275 polymorphism CC of PTGS2 was reported to be associated with a lower risk to present G3 EC [8]. Moreover, with the widespread of fertility sparing surgery that can be proposed only in case of type $1 \mathrm{G} 1$ EC at stage 1 [26], to know that the risk of G2-G3 EC is genetically reduced might be reassuring [8].

The strength of our study concern the selection of a very homogeneous population of type I EC patients who received an upfront surgery with adequate management and follow-up length. The centralization of $\mathrm{ABO}$ assessment, of treatment, of follow- up, and of pathology review are further study strengths that ensured a uniformity of treatment, of staging procedures, post-treatment monitoring, and of histological classification [8]. Instead, in other available studies the treatment and follow-up protocols widely varied $[46,47]$. Moreover, our study has also important limitations. Firstly, it had a retrospective design, and the potential and related biases/confounders are well known. Secondly, it might be underpowered due to the small cohort studied, and current sample size might not be sufficient to detect a synergistic effect in a replicate study, moreover this finding could be limited to the ethnicity.

In conclusion, current preliminary analysis demonstrates that the differentiation of the type I EC may be significantly and independently influenced by $\mathrm{ABO}$ blood group.

Patients with A genotype have a lower risk to develop G3 EC. If our results will be confirmed in large multicenter studies, $\mathrm{ABO}$ blood group might represent an useful, easy access and cheap biomarker for patients' selection and for management personalization of type I EC patients. 


\section{Competing Interests}

The authors have declared that no competing interest exists.

\section{References}

1. Bakkum-Gamez JN, Gonzalez-Bosquet J, Laack NN, Mariani A, Dowdy SC. Current issues in the management of endometrial cancer. Mayo Clin Proc. 2008; 83: 97-112.

2. Torre LA, Bray F, Siegel RL, Ferlay J, Lortet-Tieulent J, Jemal A. Global cancer statistics, 2012. CA Cancer J Clin. 2015; 65: 87-108.

3. Amant F, Moerman P, Neven P, Timmerman D, Van Limbergen E, Vergote I. Endometrial cancer. Lancet 2005; 366: 491-505.

4. Bray F, Loos AH, Oostindier M, Weiderpass E. Geographic and temporal variations in cancer of the corpus uteri: incidence and mortality in pre- and postmenopausal women in Europe. Int J Cancer 2005; 117: 123-31.

5. Needleman P, Turk J, Jakschik BA, Morrison AR, Lefkowith JB. Arachidonic acid metabolism. Annu Rev Biochem. 1986; 55: 69-102.

6. Ohno S, Ohno Y, Suzuki N, et al. Multiple roles of cyclooxygenase-2 in endometrial cancer. Anticancer Res. 2005; 25: 3679-87.

7. Ohno S, Ohno Y, Suzuki N, Soma G, Inoue M. Cyclooxygenase-2 expression correlates with apoptosis and angiogenesis in endometrial cancer tissue. Anticancer Res. 2007; 27: 3765-3770.

8. Torricelli F, Mandato VD, Farnetti E, et al. Polymorphisms in cyclooxygenase-2 gene in endometrial cancer patients. Tumour Biol. 2015; 36: 7423-30.

9. Mandato VD, Farnetti E, Torricelli F, et al. HNF1B polymorphism influences the prognosis of endometrial cancer patients: a cohort study. BMC Cancer 2015; 15: 229.

10. Watkins WM. Biochemistry and genetics of the ABO, Lewis, and P blood group systems. In: Harris H, Hirschhorn K, edtors. Advances in human genetics. New York: Plenum Press; 1980: 1-136.

11. Ravn V, Dabelsteen E. Tissue distribution of histo-blood group antigens. APMIS. 2000; 108: 1-28.

12. Szulman AE. The histological distribution of the blood group substances in man as disclosed by immunofluorescence. IV. The ABH antigens in embryos at the fifth week post fertilization. Hum Pathol. 1971; 2: 575- 85.

13. Ito NHT. Histological and Cytochemical Localization of Blood Group Antigens. New York: Gustav Fischer Verlag/VCH Publishers, 1992: 1-85. 16, 17.

14. Watkins WM. Blood-group substances. Science 1966;152:172-81.

15. Greenwell I. Blood group antigens: molecules seeking a function? Glycoconj J 1997; 14: 159-73.

16. Clausen $\mathrm{H}$, Hakomori $\mathrm{S}$. $\mathrm{ABH}$ and related histo-blood group antigens; immunochemical differences in carrier isotypes and their distribution. Vox Sang 1989; 56: 1-20.

17. Kouri M, Nordling S, Kuusela P, Pyrhonen S. Poor prognosis associated with elevated serum CA 19-9 level in advanced colorectal carcinoma, independent of DNA ploidy or SPE. Eur J Cancer 1993; 29A: 1691-6.

18. Ravn V, Mandel U, Svenstrup B, Dabelsteen E. Blood-group NB-defined glycosyltransferase and A/B blood-group antigens in human normal and malignant endometrium in relation to morphology, age and oestrogen levels. Glycosylation \& Disease 1994; 1: 153-63.

19. Ravn V, Teglbjierg CS, Mandel U, Dabelsteen E. The distribution of type 1 chain $\mathrm{ABO}$ and related histoblood group antigens in normal cycling endometrium. Int J Gynecol Pathol. 1993; 12: 70-9.

20. Zhu ZM, Kojima N, Stroud MR, Hakomori SI, Fenderson BA. Monoclonal antibody directed to $\mathrm{Le}(\mathrm{Y})$ oligosaccharide inhibits implantation in the mouse. Biol Reprod. 1995; 52: 903-12.

21. Nakashidze, I, Diasamidze A, Baratashvili D, et al. Alteration of Sex and Non-Sex Hormones and Distribution Features of Blood ABO System Groups among the Women with Uterine Body Tumors. Journal of Cancer Therapy 2014; 5: 411-419.

22. Yuzhalin AE, Kutikhin AG. ABO and Rh blood groups in relation to ovarian, endometrial and cervical cancer risk among the population of South-East Siberia. Asian Pac J Cancer Prev. 2012; 13: 5091-6.

23. Von Elm E, Altman DG, Egger M, et al. The Strengthening the Reporting of Observational Studies in Epidemiology (STROBE) Statement: guidelines for reporting observational studies. PLoS Med. 2007. e296 doi: 10.1371/journal.pmed.0040296.

24. Mandato VD, Formisano D, Pirillo D, et al. Province wide clinical governance network for clinical audit for quality improvement in endometrial cancer management. Int J Gynecol Cancer 2012; 22: 94-100.

25. Colombo N, Preti E, Landoni F, et al. ESMO Guidelines Working Group. Endometrial cancer: ESMO Clinical Practice Guidelines for diagnosis, treatment and follow-up. Ann Oncol. 2011; 22: vi35-9.

26. Palomba S, Mandato VD, La Sala GB. New Surgical Approaches to Early-Stage Endometrial Cancer. In: Diaz-Padilla I and Garcia-Donas J, eds. Endometrial Cancer: A Comprehensive Clinical and Translational Update. New York: Nova Science Publishers; 2014: 117-146.

27. Lee JC, Chang JG. ABO genotyping by polymerase chain reaction. J Forensic Sci. 1992; 37: 1269-75.
28. Poulter M, Kemp TJ, Carritt B. DNA-based rhesus typing: simultaneous determination of RHC and RHD status using the polymerase chain reaction. Vox Sang. 1996; 70: 164-8.

29. Marinaccio M, Traversa A, Carioggia E, et al. Blood groups of the ABO system and survival rate in gynecologic tumors. Minerva Ginecol. 1995; 47: 69-76.

30. Xu WH, Zheng $\mathrm{W}$, Xiang $\mathrm{YB}$, Shu $\mathrm{XO}$. ABO blood type is associated with endometrial cancer risk in Chinese women. Chin J Cancer. 2011; 30: 766-71

31. Wolpin BM, Chan AT, Hartge P, et al. ABO blood group and the risk of pancreatic cancer. J Natl Cancer Inst. 2009; 101: 424-431

32. Melzer D, Perry JR, Hernandez D et al: A genome-wide association study identifies protein quantitative trait loci (pQTLs). PLoS Genet. 2008; 4: e1000072.

33. Hakomori S. Antigen structure and genetic basis of histo-blood groups A, B and O: their changes associated with human cancer. Biochim Biophys Acta 1999; 1473: 247.

34. Duan YF, Zhu F, Li XD, et al. Association between ABO gene polymorphism (rs505922) and cancer risk: a meta-analysis. Tumour Biol. 2015; 36: 5081-7.

35. Yazer MH. What a difference 2 nucleotides make: a short review of $\mathrm{ABO}$ genetics. Transfus Med Rev. 2005; 19: 200.

36. Dabelsteen E. ABO blood group antigens in oral mucosa. What is new? J Oral Pathol Med. 2002; 31: 65-70

37. Mandel U, Langkilde NC, Orntoft TF, et al. Expression of histo-bloodgroup-A/B-gene-defined glycosyltransferases in normal and malignant epithelia: correlation with A/B-carbohydrate expression. Int J Cancer 1992: 52: 7-12

38. Gao S, Worm J, Guldberg P, et al. Genetic and epigenetic alterations of the blood group $\mathrm{ABO}$ gene in oral squamous cell carcinoma. Int J Cancer 2004; 109: 230.

39. Hakomori S. Glycosylation defining cancer malignancy: new wine in an old bottle. Proc Natl Acad Sci. USA 2002; 99: 10231

40. Kuemmel A, Single K, Bittinger F, et al. The prognostic impact of blood group-related antigen Lewis $\mathrm{Y}$ and the $\mathrm{ABH}$ blood groups in resected non-small cell lung cancer. Tumour Biol. 2007; 28: 340-9.

41. Ichikawa D, Handa K, Withers DA, Hakomori S. Histo-blood group A/B versus $\mathrm{H}$ status of human carcinoma cells as correlated with haptotactic cell motility: approach with A and B gene transfection. Cancer Res. 1997; 57: 30926.

42. Kominato $Y$, Hata $Y$, Takizawa $H$, Tsuchiya $T$, Tsukada J, Yamamoto $F$. Expression of human histo-blood group ABO genes is dependent upon DNA methylation of the promoter region. J Biol Chem. 1999; 274: 37240 -50.

43. Kominato $\mathrm{Y}$, Hata $\mathrm{Y}$, Takizawa $\mathrm{H}$, et al. Alternative promoter identified between a hypermethylated upstream region of repetitive elements and a CpG island in human ABO histo-blood group genes. J Biol Chem. 2002; 277: 37936-48.

44. Skovlund VR. ABH and related histo-blood group antigens in normal \& malignant human endometrium in relation to genetic and hormonal factors. APMIS. Suppl.1997; 69: 1-33.

45. Le Pendu J, Marionneau S, Cailleau-Thomas A, Rocher J, Le Moullac-Vaidye B, Clément $\mathrm{M}$. ABH and Lewis histo-blood group antigens in cancer. APMIS. 2001; 109: 9-31

46. Matise TC, Ambite JL, Buyske S, et al: The Next PAGE in understanding complex traits: design for the analysis of Population Architecture Using Genetics and Epidemiology (PAGE) Study. Am J Epidemiol. 2011; 174: 849-59.

47. Diaz-Padilla I, Amir E, Marsh S, Liu G, Mackay H: Genetic polymorphisms as predictive and prognostic biomarkers in gynecological cancers: a systematic review. Gynecol Oncol. 2012; 124: 354-65 